International Journal of Modern Physics D

(C) World Scientific Publishing Company

\title{
Cosmological test with the QSO Hubble diagram
}

\author{
M. López-Corredoira ${ }^{1,2}$, F. Melia ${ }^{3}$, E. Lusso ${ }^{4}$ and G. Risaliti ${ }^{5,4}$ \\ 1 Instituto de Astrofísica de Canarias, \\ E-38205 La Laguna, Tenerife, Spain \\ 2 Departamento de Astrofísica, Universidad de La Laguna, \\ E-38206 La Laguna, Tenerife, Spain; \\ 3 Department of Physics, The Applied Math Program, and Department of Astronomy, The \\ University of Arizona, AZ 85721, USA; \\ 4 INAF - Arcetri Astrophysical Observatory, Largo E. Fermi 5, I-50125 Firenze, Italy; \\ ${ }^{5}$ Dipartimento di Fisica e Astronomia, Università di Firenze, via G. Sansone 1, 50019 Sesto
} Fiorentino (Firenze), Italy

Received Day Month Year Revised Day Month Year Communicated by Managing Editor

\begin{abstract}
A Hubble diagram (HD) has recently been constructed in the redshift range $0 \lesssim z \lesssim 6.5$ using a non-linear relation between the ultraviolet and X-ray luminosities of QSOs. The Type Ia SN HD has already provided a high-precision test of cosmological models, but the fact that the QSO distribution extends well beyond the supernova range $(z \lesssim 1.8)$, in principle provides us with an important complementary diagnostic whose significantly greater leverage in $z$ can impose tighter constraints on the distance versus redshift relationship. In this paper, we therefore perform an independent test of nine different cosmological models, among which six are expanding, while three are static. Many of these are disfavoured by other kinds of observations (including the aforementioned Type Ia $\mathrm{SNe}$ ). We wish to examine whether the QSO HD confirms or rejects these earlier conclusions. We find that four of these models (Einstein-de Sitter, the Milne universe, the Static Universe with simple tired light and the Static universe with plasma tired light) are excluded at the $>99 \%$ C.L. The Quasi-Steady State Model is excluded at $>95 \%$ C.L. The remaining four models $\left(\Lambda \mathrm{CDM} / w \mathrm{CDM}\right.$, the $R_{\mathrm{h}}=c t$ Universe, the Friedmann open universe and a Static universe with a linear Hubble law) all pass the test. However, only $\Lambda \mathrm{CDM} / w \mathrm{CDM}$ and $R_{\mathrm{h}}=c t$ also pass the Alcock-Paczyński (AP) test. The optimized parameters in $\Lambda \mathrm{CDM} / w \mathrm{CDM}$ are $\Omega_{m}=0.20_{-0.20}^{+0.24}$ and $w_{d e}=-1.2_{-\infty}^{+1.6}$ (the dark-energy equation-of-state). Combined with the AP test, these values become $\Omega_{m}=0.38_{-0.19}^{+0.20}$ and $w_{d e}=-0.28_{-0.40}^{+0.52}$. But whereas this optimization of parameters in $\Lambda \mathrm{CDM} / w \mathrm{CDM}$ creates some tension with their concordance values, the $R_{\mathrm{h}}=c t$ Universe has the advantage of fitting the QSO and AP data without any free parameters.
\end{abstract}

Keywords: cosmology: cosmological parameters - cosmology: distance scale-cosmology: observations - quasars: general

\section{Introduction}

Cosmological models with a geometry different from that in the current standard model have fallen out of favour and are rarely considered in ongoing tests using 
the latest high-precision measurements. However, even within the framework of the standard model, not all the data fit together tension free. At least some controversy still surrounds the interpretation of various measurements, and other competing models often fit at least some of these observations better than the concordance model does. ${ }^{1}$ It is therefore useful to re-examine how these alternative scenarios fare compared to $\Lambda \mathrm{CDM}$ when new, improved data become available. The principal reason is that if the latest observations strongly confirm the reasons they were disfavoured in the first place, this can only solidify the concordance model's status as the correct model of the Universe. In addition, there is the possibility that something may have been missed.

The cosmological measurements that shed light on the geometry of the Universe may be separated into two principal categories. The first includes a measurement of the fluctuations in the Cosmic Microwave Background Radiation (CMB) and the analysis of large-scale structure via the inferred distribution of galaxies. CMB anisotropies provide the most evident support for the concordance $(\Lambda \mathrm{CDM})$ model, but one should find an independent confirmation of this theory and its parameters because CMB anisotropies may be generated/modified by mechanisms other than those in the standard picture, ${ }^{2,3,4,5}$ and may also have some contamination. ${ }^{6}$

Secondly, cosmological tests using surveys of galaxies have also been developed to provide information on the geometry of the Universe. These include the angularsize test; ${ }^{7,8}$ the surface brightness (known as the 'Tolman' ${ }^{9,10}$ ) test; the use of Hubble diagrams (HD) for galaxies; ${ }^{11}$; and Gamma-Ray Bursts. ${ }^{12,13,14}$ However, these depend on the evolution of the sources, so the results of this type of test may vary hugely depending on one's interpretation. A better prospect is obtained with the Alcock-Paczyński test, ${ }^{15,16}$ which can test the geometry of the Universe independently of the evolution of galaxies.

Another good prospect is based on the use of an HD constructed from Type Ia Supernovae (SNIa) embedded within the galaxies, ${ }^{17}$ provided that we assume zero evolution and negligible extinction or selection effects (which are not universally accepted; see, e.g., criticisms by Refs. ${ }^{18,19,20}$ ). Even then, these events are detectable only up to redshift $z \sim 2 .^{21}$

Recently, a new method was presented ${ }^{22}$ of sampling the redshift-distance relationship, based on a non-linear correlation between the ultraviolet and X-ray luminosities of Quasi Stellar Objects (QSOs). This relationship appears to be independent of evolution, so suitable sources may be found out to a redshift of six. This new diagnostic was used by these authors to optimize the parameters in the standard model. In this letter, we present a followup application, based on the same data described in Ref. ${ }^{22}$, to carry out a comparative analysis of nine different cosmological models, six of them with expansion and three representing a static Universe. Exotic models invoking a static Universe are included for the simple reason that the discussion on the reality of the expansion is still being discussed within some literature. ${ }^{23}$ The principal reason for including them here is not to resurrect them but, rather, to make use of this excellent new diagnostic tool to re-test them against the standard 
model independently of previous studies. In principle, since this diagnostic is based on a distribution of sources extending over a much larger range in redshift than, say, the Type Ia SNe, it has the potential of providing tighter constraints than are currently available. Such an independent test should be viewed as a complement to current consensus, in the sense that if this test also disfavours models already disfavoured by other observations, then their case is further weakened in comparison to models that are favoured.

\section{Competing cosmological scenarios}

We will test nine different cosmological models, each with its unique expression for the luminosity distance, $d_{L}(z)$. We assume a Hubble constant $H_{0}=70 \mathrm{~km} \mathrm{~s}^{-1}$ $\mathrm{Mpc}^{-1}$ throughout.

(1) The flat (concordance) $\Lambda$ CDM model, characterized by the parameters $\Omega_{m}=$ $0.3, \Omega_{\Lambda}=0.7$ and a dark-energy equation-of-state $w_{\Lambda}=-1$. Here, $\Omega_{i}$ is the energy density $\rho_{i}$ of species $i$, scaled to today's critical density, $\rho_{c} \equiv 3 c^{2} H_{0}^{2} / 8 \pi G$. In this model,

$$
d_{L}(z)=\frac{c}{H_{0}}(1+z) \int_{0}^{z} \frac{d u}{\sqrt{\Omega_{m}(1+u)^{3}+\Omega_{\Lambda}(1+u)^{3\left(1+w_{\Lambda}\right)}}} .
$$

(2) Einstein-de Sitter (essentially Eq. 1 with $\Omega_{m}=1$ and $\Omega_{\Lambda}=0$ ):

$$
d_{L}(z)=2 \frac{c}{H_{0}}(1+z)\left(1-\frac{1}{\sqrt{1+z}}\right) .
$$

Although this is no longer the standard model, some researchers still view it as more appropriate than the concordance model. ${ }^{24,25}$

(3) A Friedmann model with negative curvature. Here, $\Omega_{m}=0.3, \Omega_{\Lambda}=0$, implying a curvature term with $\Omega_{K}=1-\Omega_{m}=0.7$ (Ref. ${ }^{26}[\S 7.4 .1]$ ):

$$
d_{L}(z)=\frac{c}{H_{0}} \frac{(1+z)}{\sqrt{\Omega_{K}}} \sinh \left(\int_{0}^{z} \frac{\sqrt{\Omega_{K}} d u}{\sqrt{\Omega_{m}(1+u)^{3}+\Omega_{K}(1+u)^{2}}}\right) .
$$

Such a model might be relevant when one wishes to avoid including a cosmological constant (i.e., $\Omega_{\Lambda}=0$ ).

(4) The Quasi-steady State Cosmology (QSSC ${ }^{2}$ ):

$$
d_{L}(z)=\frac{c}{H_{0}}(1+z) \int_{0}^{z} \frac{d u}{\sqrt{\Omega_{c}(1+u)^{4}+\Omega_{m}(1+u)^{3}+\Omega_{\Lambda}}} .
$$

This cosmology is not the standard model, but has been used to fit an assortment of HDs, e.g., for SNIa. ${ }^{27,28,29}$ The expansion with an oscillatory term produces a dependence of the luminosity and angular-diameter distances similar to those of the standard model, though adding the effects of matter creation (the so-called C-field, for which $\Omega_{c}=1-\Omega_{m}-\Omega_{\Lambda}$ ) with slight variations depending on the parameters, which are not as well constrained as those in the 
standard model. Here, we will keep $\Omega_{m}$ and $\Omega_{\Lambda}$ as unconstrained parameters. Previous estimates of these variables imply that galaxies should only be observable out to a maximum redshift of $z \lesssim 6$, a result that is already incompatible with galaxies observed at redshift 8 and beyond. ${ }^{30}$

(5) The $R_{\mathrm{h}}=c t$ Universe (a Friedmann-Robertson-Walker cosmology with zero active mass). This model has a total equation-of-state $\rho+3 p=0$, where $\rho$ and $p$ are, respectively, the total energy density and pressure of the cosmic fluid. ${ }^{31,32,33}$ In this cosmology,

$$
d_{L}(z)=\frac{c}{H_{0}}(1+z) \ln (1+z) .
$$

(6) The Milne Universe. This solution may be obtained from the more generic FRW equations by demanding that the energy density, pressure and cosmological constant are all equal to zero and the spatial curvature is negative $(k=-1)$. From these assumptions, and the Friedmann equations, it follows that the scale factor must depend linearly on time. In the model, the mathematical equivalence of the zero energy density $(\rho=0)$ version of the FRW metric with Milne's model implies that a full general relativistic treatment using Milne's assumptions would result in an increasing scale factor and associated metric expansion of space, with the feature of a linearly increasing scale factor for all time. ${ }^{34,35}$. The luminosity distance is ${ }^{34}$

$$
d_{L}(z)=\frac{c}{H_{0}}(1+z) \sinh [\ln (1+z)] .
$$

(7) A static Euclidean model with a linear Hubble law at all redshifts:

$$
d_{L}(z)=\frac{c}{H_{0}} \sqrt{1+z} z .
$$

This model has been used by some authors to account for certain specific observations. ${ }^{10}$ This scenario assumes that the Universe is static. The factor $\sqrt{1+z}$ stems from the loss of energy due to a redshift without expansion. This factor is different from $(1+z)$ because there is no time dilation. The challenge with this model is to account for the redshift using a mechanism different from the conventional expansion/Doppler effect. This cosmology has not been explored theoretically and/or mathematically, but its promoters argue that, from a phenomenological point of view, one may consider this relationship between distance and redshift as an extrapolation of the observed behavior at low redshifts. In this paper, our goal is simply to test its predictions against the QSO data, independently of how or why one may justify its theoretical basis.

(8) A static Euclidean model with tired light:

$$
d_{L}(z)=\frac{c}{H_{0}} \sqrt{1+z} \ln (1+z)
$$


This phenomenological representation stems from the idea that photons lose energy along their trajectory due to some interaction, and the relative loss of energy is proportional to the path length i.e., $\frac{d E}{d r}=-\frac{H_{0}}{c} E \cdot{ }^{36}(\S 7.3)$. Of course, as in the previous case, this ansatz does not enjoy much support among cosmologists, but our goal here is merely to test its predictions agains the QSO data.

(9) A static Euclidean model with plasma tired light:

$$
d_{L}(z)=\frac{c}{H_{0}}(1+z)^{3 / 2} \ln (1+z) .
$$

The plasma redshift application ${ }^{37}(\S 5.8)$ introduces a factor $(1+z)^{3 / 2}$ instead of $(1+z)^{1 / 2}$ to take into account an additional Compton scattering which is double that of the plasma redshift absorption.

\section{The QSO HD}

A nonlinear relation between the rest-frame ultraviolet $(2500 \AA)$ and X-ray $(2 \mathrm{keV})$ luminosities of quasars, of the type $\log L_{X}=\beta+\gamma \log L_{\mathrm{UV}}$ (e.g. Refs. ${ }^{38,39,40}$ ), is what allows to derive a Hubble diagram for these sources. Equation (5) in Ref. ${ }^{22}$ relates the rest-frame ultraviolet and X-ray fluxes of QSOs according to

$$
D M(z)=\frac{5}{2(\gamma-1)}\left[\log _{10}\left(F_{\mathrm{X}}\right)-\gamma \log _{10}\left(F_{\mathrm{UV}}\right)-\beta^{\prime}\right],
$$

where $\gamma$ and $\beta^{\prime}\left(=\beta+(\gamma-1) \log _{10}(4 \pi)\right.$ are constants (in principle independent of $z$ if there is no evolution), and

$$
D M(z) \equiv 5 \log _{10}\left[d_{L}(z / \mathrm{Mpc})\right]-25
$$

is the distance modulus. In their analysis, Ref. ${ }^{22}(\S 4)$ also determined that $\gamma=$ $0.60 \pm 0.02$ for all $z$, and this result is independent of the cosmological model since it only depends on the fluxes at rest that are derived from the observations. Hence,

$$
D M(z)+A=-(6.25 \pm 0.31)\left[\log _{10}\left(F_{\mathrm{X}}\right)-(0.60 \pm 0.02) \log _{10}\left(F_{\mathrm{UV}}\right)\right],
$$

where $A$ is an arbitrary scaling factor. This is the relationship that we will use to examine how well the expressions for $d_{L}$ predicted by the various cosmologies introduced in $\S 2$ fit the data.

For this purpose, we adopt the data prepared by Ref. ${ }^{22}$ and bin them in a weighted average in intervals of $\Delta \log _{10} z=0.1$, ensuring that there are $N \geq 4$ QSOs/bin. In total, we have 18 data points with averaged values of $-6.25\left[\log _{10}\left(F_{\mathrm{X}}\right)-0.60 \log _{10}\left(F_{\mathrm{UV}}\right)\right]$, and an error given by $\frac{r . m . s .}{\sqrt{N-1}}$ (see Fig. 1). Using the dispersion of values to determine the error of the average is more accurate than using the individual error bars for the fluxes from different sources that are underestimated or unknown in some cases. As argued by Ref. ${ }^{22}$, the method chosen to bin the data does not significantly affect the fits. 
Table 1. Results of the $\chi^{2}$ test, best-fit free cosmological parameters, if any ( $\nu$ is the number of free parameters), and associated probabilities. The number of points is $N=18$. The model number corresponds to the list in $\S 2$.

\begin{tabular}{lcclcc}
\multicolumn{1}{c}{ Model } & $\nu$ & $\chi_{\text {dof,min }}^{2}$ & Free cosmol. parameters & $A$ & Probability \\
\hline (1)- $\Lambda$ CDM $\Omega_{m}=0.3, w_{\Lambda}=-1$ & 1 & 1.26 & - & 50.21 & 0.21 \\
(1)- $\Lambda$ CDM $w_{\Lambda}=-1, \Omega_{m}$ free & 2 & 1.27 & $\Omega_{m}=0.19_{-0.11}^{+0.20}$ & 50.01 & 0.21 \\
(1)-wCDM both $\Omega_{m}, w_{\text {de }}$ free & 3 & 1.35 & $\Omega_{m}=0.20_{-0.20}^{+0.24}, w_{d e}=-1.2_{-\infty}^{+1.6}$ & 49.94 & 0.16 \\
(2)-EdS & 1 & 1.98 & - & 50.89 & $9.1 \times 10^{-3}$ \\
(3)-Fr.neg.curv. $\Omega_{m}=0.3$ & 1 & 1.41 & - & 50.39 & 0.12 \\
(3)-Fr.neg.curv. $\Omega_{m}$ free & 2 & 1.49 & $\Omega_{m}=0.35_{-0.20}^{+0.28}$ & 50.44 & 0.093 \\
(4)-QSSC $\Omega_{m}, \Omega_{\Lambda} \leq 0$ free & 3 & 2.01 & $\Omega_{m}=1.17_{-0.15}^{+0.42}, \Omega_{\Lambda}=-0.01_{-0.36}^{+0.01}$ & 50.77 & 0.011 \\
(5)- $R_{h}=c t$ & 1 & 1.38 & - & 50.40 & 0.14 \\
(6)-Milne & 1 & 2.23 & - & 50.04 & $2.5 \times 10^{-3}$ \\
(7)-St.lin.Hub. & 1 & 1.45 & - & 50.31 & 0.10 \\
(8)-St.tir.l. & 1 & 3.82 & - & 51.42 & $1.6 \times 10^{-7}$ \\
(9)-St.pl.tir.l. & 1 & 3.23 & - & 49.38 & $7.1 \times 10^{-6}$ \\
\hline
\end{tabular}




\subsection{QSO HD on its own}

Table 3 and Fig. 1 show the results of our fits to the QSO data using the nine cosmologies introduced in $\S 2$. Some of these models have fixed parameters; others have parameters that need to be optimized in producing the best fits. Of the nine cases, five are excluded at the $>95 \%$ C.L.. These are Einstein-de Sitter, the Quasisteady State, the Milne Universe, the Static Euclidean model with simple tired light, and the Static Euclidean model with plasma tired light. All of them, except Quasistady State, are excluded at the $>99 \%$ C.L. The remaining four models (standard $\Lambda \mathrm{CDM}, R_{\mathrm{h}}=c t$, the Friedmann open universe, and the Static model with a linear Hubble law) pass the test. If we optimize $\Omega_{m}$ in $\Lambda$ CDM, the best fit is obtained with $\Omega_{m}=0.19_{-0.11}^{+0.20}$ ( $1 \sigma$ error bars); and if we allow both $\Omega_{m}$ and $w_{d e}$ to be free, then the best fit corresponds to the optimized parameter values $\Omega_{m}=0.20_{-0.20}^{+0.24}$, $w_{d e}=-1.2_{-\infty}^{+1.6}$. The error bars include the uncertainty in $\gamma$ (one of the constants in Eq. 10). 

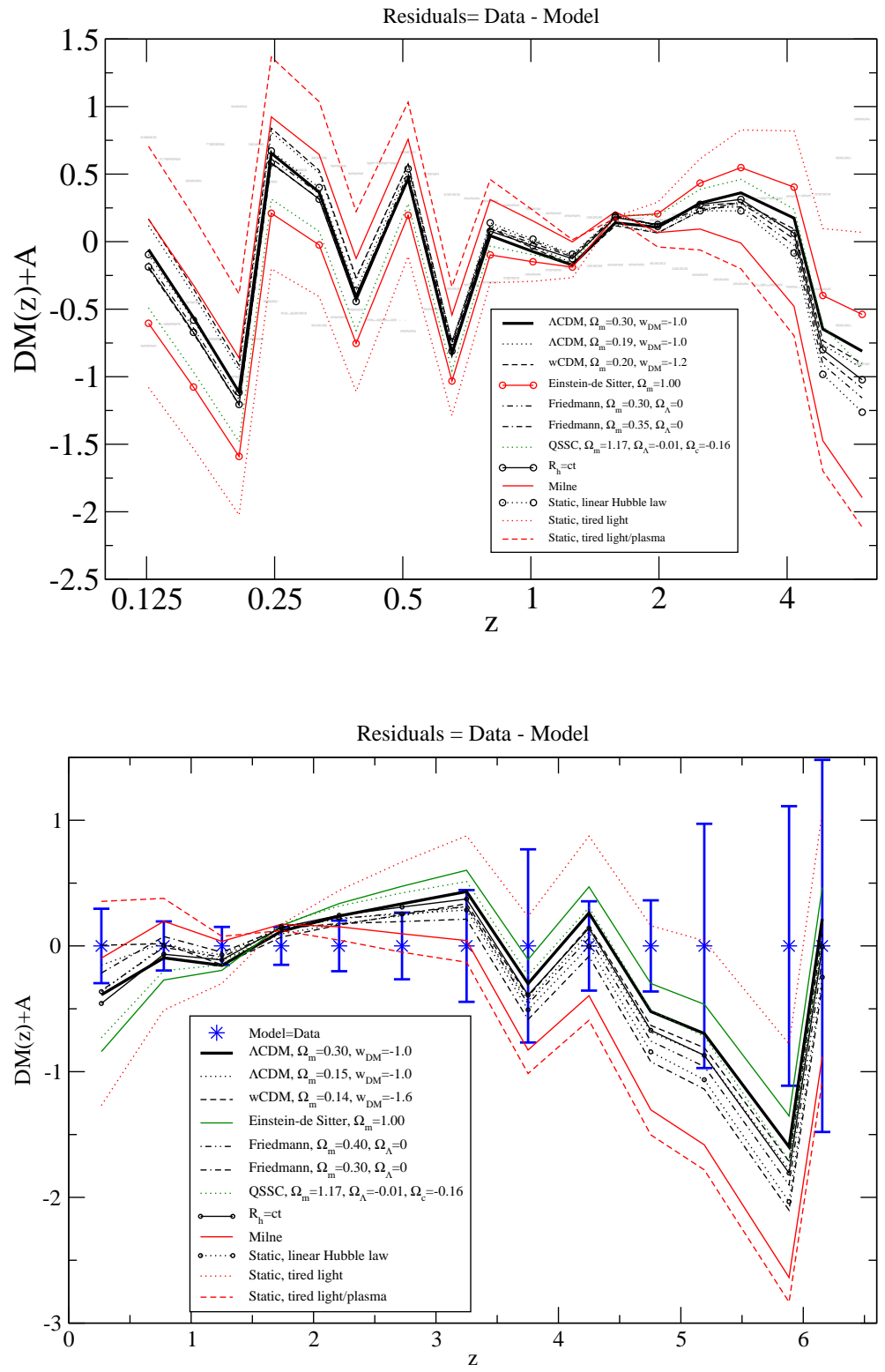

Fig. 1. Top: Log-linear plot of data and best fits for the distance modulus (+scaling constant A) in various cosmological models (see Table 3). Bottom: residuals (Data-Model) corresponding to the above fits, for which the grey shaded region is the region within the error bar for which Data and Model are coincident. Black color indicates that the fit is good within 95\% C.L.; green color indicates that the cosmological model is excluded at a C.L. between $95 \%$ and $99 \%$; red color indicates that the cosmological model is excluded at C.L. larger than $99 \%$. Error bars only reflect the error of the average due to the dispersion of data; they do not contain the error in $\gamma$, which is fixed at $\gamma=0.60$. 
Table 2. Combined QSO HD + Alcock-Paczynski (AP) test. References for the AP results are: Refs. ${ }^{15,16}$. For the Milne model, the results of the AP test were not published, but we have calculated the probability here using the same method and

\begin{tabular}{llccc}
\multicolumn{1}{c}{ Model } & \multicolumn{2}{c}{ data as in Ref. ${ }^{16}$. This reference is denoted Ref. ${ }^{16 *}$. } & & \\
& Probability AP & Source AP & Combined probability \\
\hline (1)- $\Lambda$ CDM $\Omega_{m}=0.3, w_{\Lambda}=-1$ & - & 0.0066 & Ref. $^{16}$ & 0.036 \\
(1)- $\Lambda$ CDM $w_{\Lambda}=-1, \Omega_{m}$ free & $\Omega_{m}=0.20_{-0.04}^{+0.06}$ & 0.0095 & Ref. $^{16}$ & 0.057 \\
(1)-wCDM $\Omega_{m}, w_{d e}$ free & $\Omega_{m}=0.38_{-0.19}^{+0.20}, w_{d e}=-0.28_{-0.40}^{+0.52}$ & - & Ref. $^{16}$ & 0.16 \\
(2)-EdS & - & $2.7 \times 10^{-4}$ & Ref. $^{15}$ & $4.1 \times 10^{-7}$ \\
(3)-Fr.neg.curv. $\Omega_{m}=0.3$ & - & 0.0034 & Ref. $^{15}$ & 0.0029 \\
(3)-Fr.neg.curv. $\Omega_{m}$ free & $\Omega_{m}=0.03_{-0.03}^{+0.02}$ & 0.024 & Ref. $^{15}$ & 0.0016 \\
(4)-QSSC $\Omega_{m}, \Omega_{\Lambda} \leq 0$ free & $\Omega_{m}=1.22_{-0.09}^{+0.11}, \Omega_{\Lambda}=0.0_{-0.08}^{+0.0}$ & 0.020 & Ref. $^{15}$ & $1.4 \times 10^{-3}$ \\
(5)- $R_{h}=c t$ & - & 0.96 & Ref. $^{16}$ & 0.21 \\
(6)-Milne & - & $1.1 \times 10^{-9}$ & Ref. $^{16 *}$ & $7.3 \times 10^{-10}$ \\
(7)-St.lin.Hub. & - & $1.4 \times 10^{-5}$ & Ref. $^{15}$ & $2.2 \times 10^{-5}$ \\
(8)-St.tir.l. & - & 0.96 & Ref. $^{16}$ & $6.0 \times 10^{-7}$ \\
(9)-St.pl.tir.l. & - & 0.96 & Ref. ${ }^{16}$ & $2.3 \times 10^{-5}$ \\
\hline
\end{tabular}




\subsection{QSO HD combined with the Alcock-Paczyński test}

An application of the Alcock-Paczyński (AP) test to cosmological models can provide additional tight constraints, ${ }^{15,16}$ independently of the present HD for QSOs. Very importantly, the AP test is entirely independent of any galaxy evolution and this is the reason for choosing it among other possible constraints using only the galaxy distribution. The reason for this independence is that the measured quantity - the ratio of observed angular size to radial/redshift size in the anisotropic twopoint correlation function - depends only on the geometry of the Universe, provided the distribution of galaxies is spherical, which is always true at any age of the Universe. The only sources of contamination in that measurement are the redshift distortions produced by the peculiar velocities of the galaxies, but there is a way to overcome them with the inclusion in the AP test of an observational signature with a sharp feature, such as the Baryonic Acoustic Oscillation (BAO) peak. ${ }^{16}$ We avoid combining these tests with the analysis of CMB data, first, because the latter has already been used by many other authors and we want to introduce new considerations that have not been adopted in previous papers; and second, because the $\mathrm{CMB}$ analysis is not free of interpretation and needs some modelling, for instance, with the foreground contamination, that makes it dependent on considerations other than a pure cosmological geometry (see §1). In Table 3.1, we list the combined total probability for the QSO HD and AP analysis (based on the most recent and most accurate baryon acoustic oscillation [BAO] measurements in the data from Ref. ${ }^{16}$ ), including the best-fit parameters resulting from a combination of the Gaussian distributions in both tests. This combined probability is calculated by summing the $\chi^{2}$ 's and the number of degrees of freedom $(D F)$ of both tests and calculating the corresponding probability of this sum, i.e.,

$$
\begin{aligned}
& P_{1+2}=P\left(\chi^{2}, D F\right) \\
& \chi^{2}=\chi_{1}^{2}\left(P_{1}, D F_{1}\right)+\chi_{2}^{2}\left(P_{2}, D F_{2}\right) \\
& D F=D F_{1}+D F_{2} .
\end{aligned}
$$

The combined test leaves only two models that are not excluded at $>95 \%$ C.L.: $\Lambda$ CDM with parameters different from the standard model and $R_{\mathrm{h}}=c t$. The $R_{\mathrm{h}}=c t$ cosmology has the advantage that it can produce a good fit without any free parameters, whereas the parameter optimization $\left(\Omega_{m}=0.38\right.$ and $\left.w_{d e}=-0.28\right)$ in $\Lambda \mathrm{CDM}$ produces some tension with the concordance values $\Omega_{m}=0.3$ and $w_{d e} \equiv$ $w_{\Lambda}=-1$ (see also Ref. ${ }^{16}$ ). The variation of cosmological parameters in $\Lambda$ CDM would thus diminish the level of concordance with other cosmological data. On the other hand, the $R_{\mathrm{h}}=c t$ Universe without any free parameters has successfully passed all other cosmological tests applied to it thus far. ${ }^{41,42,43,44,45,13,46,47}$

\section{Discussion and Conclusion}

A HD diagram for QSOs can constrain cosmological models in ways that many other tests, e.g., involving the use of Type Ia SNe, cannot, since quasar spectra can 
be studied at redshifts which are not accesible for Type Ia SNe. Unfortunately, the present sample of objects that may be used for this study still results in distance moduli with a large dispersion at high $z$, roughly of order one magnitude, that mitigates the overall power of this test. Furthermore, this test assumes that the relation between the X-ray and UV fluxes is independent of redshift, which is reinforced by the good fits produced assuming a constant $\gamma(z)=0.60$ for $z \lesssim 6 .^{22}$ Nonetheless, a $\gamma(z)$ with small variations in redshift is not excluded within the current error bars.

Another caveat in this analysis is that there may be some unaccounted for systematics associated with the determination of the K-corrections used to calculate the X-ray flux in the rest frame (Ref. ${ }^{22}, \S$ A.5). Nonetheless, provided that these systematics and any possible evolution in the relation between the X-ray and UV fluxes are negligible, one can use present-day data to check whether a cosmological model predicts a distance modulus in line with the QSO observations over a wide range in redshift.

Ref. ${ }^{22}$ was able to constrain the cosmological parameters in the standard model with the available data. With these same data, we demonstrated in this letter that five of nine different cosmological models can be excluded at $>95 \%$ C.L. These models are: the Quasi-steady State model, Einstein-de Sitter, the Milne Universe, the Static Euclidean model with simple tired light and the Static Euclidean model with plasma tired light. These last four are excluded very strongly, at $>99 \%$ C.L.

The remaining four models [standard $\Lambda \mathrm{CDM}, R_{\mathrm{h}}=c t$ (with zero active mass), the Friedmann open model and the Static model with a linear Hubble law] all pass the QSO HD test, but only the first two also pass the Alcock-Paczyński test using the latest, high-precision BAO data. Future surveys will increase the QSO sample suitable for this study and permit a determination of the X-ray K-correction directly from their spectra, with the possibility of further increasing the precision of the QSO $\mathrm{HD}$ and eliminating additional models from the list in $\S 2$. At the same time, this diagnostic will continue to refine the optimization of parameters in the standard model.

\section{Acknowledgments}

Thanks are given to the anonymous referee for helpful comments. FM is grateful to Amherst College for partial support through the John Woodruff Simpson Chair. EL and GR have been supported by the grant PRIN-INAF 2012.

\section{References}

1. M. López-Corredoira, Studies in History and Philosophy of Modern Physics 46-A (2014) 86.

2. J. V. Narlikar, G. Burbidge and R. G. Vishwakarma, J. Astrophys. Astron. 28 (2007) 67.

3. G. W. Angus and A. Diaferio, Mon. Not. R. Astron. Soc. 417 (2011) 941.

4. M. López-Corredoira, Int. J. Mod. Phys. D 22 (2013) id. 1350032.

5. F. Melia, Astron. Astrophys. 561 (2014) id. A80. 
6. M. López-Corredoira, J. Astrophys. Astron. 28 (2007) 101.

7. K. P. Kapahi, in Observational Cosmology (IAU Symp. 124), eds. A. Hewitt, G. Burbidge and L. Z. Fang (Reidel, Dordrecht, 1987), p. 251.

8. M. López-Corredoira, Int. J. Mod. Phys. D 19 (2010) 245.

9. L. M. Lubin and A. Sandage, Astron. J. 122 (2001) 1084.

10. E. J. Lerner, R. Falomo and R. Scarpa, Int. J. Mod. Phys. D 23 (2014) id. 1450058.

11. D. Schade, L. F. Barrientos and O. López-Cruz, Astrophys. J. Lett. 477 (1997) L17.

12. H. Wei, J. Cosmol. Astropart. Phys. 8 (2010) 20.

13. J.-J. Wei, X. Wu and F. Melia, Astrophys. J. 772 (2013) 43.

14. J.J. Wei et al., Mon. Not. R. Astron. Soc. 439 (2014) 3329.

15. M. López-Corredoira, Astrophys. J 781 (2014) 96.

16. F. Melia and M. López-Corredoira, arXiv:1503.05052 (2015).

17. M. Kowalski et al., Astrophys. J. 686 (2008) 749.

18. L. G. Balázs et al., Astron. Nachrichten 327 (2006) 917.

19. P. Podsiadlowski et al., New Astron. Rev. 52 (2008) 381.

20. A. I. Bogomazov and A. V. Tutukov, Astron. Reports 55 (2011) 497.

21. D. O. Jones et al., Astrophys. J. 768 (2013) id. 166.

22. G. Risaliti and E. Lusso, Astrophys. J. 815 (2015) 33.

23. M. López-Corredoira, Proceedings of Science (SISSA) (FFP14)085 (2014) [arXiv:1501.01487].

24. S. C. Vauclair et al., Astron. Astrophys. 412 (2003) L37.

25. A. Blanchard, in: Current issues in Cosmology, eds. J.-C. Pecker and J. V. Narlikar, (Cambridge University Press, Cambridge (U.K.), 2006), p. 76.

26. Y. Baryshev and P. Teerikorpi, Fundamental Questions of Practical Cosmology (Springer, Dordrecht, 2012).

27. S. K. Banerjee et al., Astron. J. 119 (2000) 2583.

28. J. V. Narlikar, R. G. Vishwakarma, R. G. and G. Burbidge, Publ. Astron. Soc. Pacific 114 (2002) 1092.

29. R. G. Vishwakarma, Mon. Not. R. Astron. Soc. 331 (2002) 776.

30. M. D. Lehnert et al., Nature 467 (2010) 940.

31. F. Melia, Mon. Not. R. Astron. Soc. 382 (2007) 1917.

32. F. Melia and A. S. H. Shevchuk, Mon. Not. R. Astron. Soc. 419 (2012) 2579.

33. F. Melia, Front. Phys. 11(4) (2016) Id. 119801 (arXiv:1601.04991).

34. R. G. Vishwakarma, Phys. Scr. 87 (2013) 055901.

35. O. I. Chashchina and Z. K. Silagadze, Universe 1 (2015) 307.

36. P. A. LaViolette, Subquantum kinetics: The Alchemy of Creation (4th ed.) (Starlane Pub., Niskayana (NY), 2012).

37. A. Brynjolfsson, arXiv:astro-ph/0401420 (2004).

38. A. T. Steffen et al., Astron. J. 131 (2006), 2826.

39. D. W. Just et al., Astrophys. J. 665 (2007), 1004

40. E. Lusso et al., Astron. Astrophys. 512 (2010), A34.

41. F. Melia, Astrophys. J. 764 (2013) 72.

42. F. Melia, Astron. Astrophys. 553 (2013) A76.

43. F. Melia, Astron. J. 147 (2014) 120.

44. F. Melia, Astron. J. 149 (2015) 6.

45. F. Melia, J.-J. Wei and X. Wu, Astron. J. 149 (2015) 2.

46. J. J. Wei, X. Wu and F. Melia, Astrophys. J. 788 (2014) 190.

47. J. J. Wei, X. Wu and F. Melia, Mon. Not. R. Astron. Soc. 447 (2015) 479. 